\title{
La integración de las nuevas tecnologías de la información y la comunicación en la escuela. Factores relevantes
}

\author{
Bernardo Gargallo López \\ bernardo.gargalloguv.es \\ Jesús Suárez Rodríguez \\ rodriguseuv.es \\ Universidad de Valencia
}

\section{El contexto. La sociedad de la información}

Los cambios operados en las tecnologías han comportado también cambios radicales en la organización del conocimiento, en los procesos cognitivos del ser humano y en la organización y prácticas sociales. Y es que la relación del hombre con la tecnología es compleja: él la crea y la utiliza para amplificar sus sentidos pero, a la vez, la propia tecnología lo transforma a su vez a él mismo y a la sociedad. En ese sentido, se puede dividir la historia del hombre en fases o períodos caracterizados por la "tecnología" dominante de codificación, almacenamiento y recuperación de la información (Adell, 1997; Bosco, 1995; Harnad, 1991; Levinson, 1990):

$1^{\text {a }}$-La aparición del lenguaje oral, que permite conservar la experiencia, acumular y almacenar la cultura y transmitirla a las nuevas generaciones (García Carrasco, Ferrer, Mompó y Naya, 1998 ; Ong, 1995), con un papel predominante de la memoria para almacenar la información.

$2^{\mathrm{a}}$.-La aparición y difusión de la escritura, que permite la independencia espacio-temporal entre emisor-receptor, libera de la inmediatez y permite la acumulación y preservación de los conocimientos para la posteridad, con algunos inconvenientes (la palabra escrita es más lenta, la audiencia es menor, es más elitista -al menos durante mucho tiempo- y es menos interactiva que el habla),ventajas evidentes (fiabilidad y sistematización que favorecieron el desarrollo de la ciencia, mayor capacidad para la reflexión, deliberación y estructuración) y nuevas necesidades (no se aprende a leer y escribir simplemente por modelado y repetición de lo que hacen los mayores, hacen falta medios extraordinarios, iniciación sistemática, escuelas...) (Adell, 1997; Bosco, 1995; García Carrasco y otros, 1998).

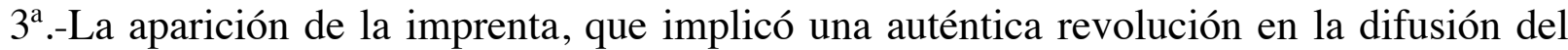
conocimiento al hacerlo accesible a la población y al eliminar el carácter elitista, que dio origen a la cultura moderna (Adell, 1997; Eisenstein, 1994).

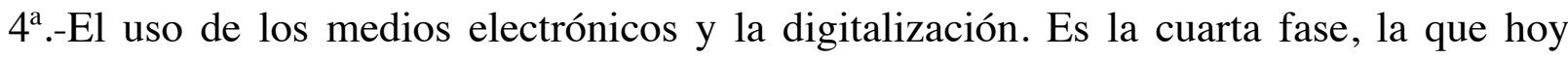
vivimos. Los avances tecnológicos han permitido crear entornos de comunicación totalmente nuevos, que no están sujetos a un medio físico y en los que la información se sitúa en un espacio no real (ciberespacio o espacio virtual), de modo que se puede transmitir la información de modo instantáneo y a nivel mundial. Las nuevas tecnologías de la información y la comunicación, entendidas como el "conjunto convergente de tecnologías de la microelectrónica, la informática (máquinas y software), las 
telecomunicaciones y la optoelectrónica" (Castells, 2000a, 60) están produciendo una auténtica revolución, con cambios importantísimos en nuestra sociedad, que se concretan en una denominación: la sociedad de la información (Adell, 1997; Bosco, 1995; García Carrasco, Ferrer, Mompó y Naya, 1998; Castells, 2000a y 2000b; Echevarría, 1994; Harnad, 1991; Joyanes, 1996; Levinson, 1990; Negroponte, 1995; Ong, 1995; Postman, 1994; Terceiro, 1996; Marina, 1999), a la que se han dado diversas denominaciones que sirven para enfatizar sus características definitorias: así, Castells (2000a) la denomina "sociedad red", Echevarría (1994) "telépolis", Joyanes (1996) "cibersociedad", Negroponte (1995) "mundo digital", Postman (1994) "tecnópolis", y Terceiro (1996) "sociedad digital".

Se trata de una nueva sociedad en que se prevé que un porcentaje importante de la masa laboral mundial trabaje en el terreno de las NTIC, que se sustentará sobre nuevas actividades relacionadas con las redes, el comercio electrónico, los hipermedia (hipertexto, multimedia y realidad virtual) y los nuevos soportes de comunicaciones (cable, satélites, etc.) (Joyanes, 1999). Estas innovaciones tecnológicas están provocando cambios sociales importantísimos en la organización del trabajo, en las relaciones empresariales, en la cultura, en la educación, etc.

Son de tan hondo calado las repercusiones que Castells (2000a) habla de un nuevo paradigma tecnoeconómico, el paradigma de la tecnología de la información, cuyos rasgos constituyen la base material de la "sociedad red":

- La información es su materia prima. Se trata de tecnologías para actuar sobre la información, no sólo información para actuar sobre la tecnología.

- La mayoría de los procesos de nuestra existencia individual y colectiva están directamente influidos por esa tecnología.

- La morfología de red parece estar bien adaptada para una complejidad de interacción creciente y dota de flexibilidad al sistema (lógica de 1 a interconexión)

- La flexibilidad y capacidad para reconfigurarse, un rasgo decisivo en una sociedad caracterizada por el cambio constante y por la fluidez organizativa.

- La convergencia e integración creciente de tecnologías específicas en un sistema altamente integrado. Así, la microelectrónica, las telecomunicaciones, la optoelectrónica y los ordenadores están ahora integrados en sistemas de información.

- Por fin, el paradigma de la tecnología de la información no evoluciona hacia su cierre como sistema, sino hacia su apertura como una red multifacética. Sus cualidades fundamentales con su carácter integrador, la complejidad y la interconexión.

Ante esta nueva realidad no cabe la indiferencia y, frente a posiciones que postulan que la nueva sociedad asentada en las nuevas tecnologías va a comportar la panacea, la solución de todos los males (Gates, 1995; Negroponte, 1995) y otras que sólo ven amenazas (Postman, 1994; Stoll, 1996), como expertos en educación y educadores debemos ser críticos -ni "apocalípticos" ni "integrados"-, y analizar los porqués, las posibilidades y las limitaciones.

Las redes informáticas constituyen, de entre todas las novedades en materia de tecnologías de la información y la comunicación, uno de los desarrollos más espectaculares de este siglo. Internet es la mayor red de ordenadores del planeta (Adell, 1998). En realidad, no es tanto una red como una red de redes, una red de varias decenas de miles de redes locales y de área amplia interconectadas (interconexión: "internet" en inglés) entre sí y que permiten compartir información, recursos y servicios. Para ello utiliza unos protocolos o acuerdos de comunicación entre ordenadores que permiten comunicarse sin restricciones (Bartolomé, 1999). 
Para hacerse una idea de las proporciones del fenómeno y del crecimiento espectacular de Internet basta con los siguientes números: en 1988 se estimaban unos 28.000 ordenadores conectados a Internet. A finales de 1995 se habían convertido en 9 millones; a finales de 2000 en 350 millones; a mediados de 2001 se calculaba que habría alrededor de 700 millones de ordenadores conectados, y entre 2005-2007 se estimaba que llegarían a 2.000 millones como mínimo (Castells, 2000b). Se trata, pues, de un fenómeno de carácter universal aunque es cierto que hay zonas en el mundo todavía muy desconectadas.

En nuestro país, Internet llegó a mediados del año 1990 (Barberá, 1995; Sanz, 1994) de la mano del Proyecto IRIS (gestionado por Fundesco y actualmente a cargo de la RedIRIS (http://www.rediris.es/), y desde entonces y especialmente en los últimos años, el crecimiento ha sido incesante. Los últimos datos disponibles de España tomados del Estudio General de Medios llevado a cabo por AIMC (Asociación para la Investigación de Medios de Comunicación) que llegan hasta mayo de 2001, revelan un crecimiento espectacular: en abril-mayo de 1997 se calculaba el número de individuos que había utilizado Internet el último mes en 919.000; en abril-mayo de 1999 la cifra subía a 2.441.000, en abril-mayo de 2000 eran ya 3.942.000 y en abril-mayo de 20017.079 .000 (http://www.aimc.es/datosegm/internetficha.html).

\section{La incidencia de las NTIC en la educación}

Las nuevas tecnologías tienen claras repercusiones sobre la educación dado que inciden en la conformación del ser humano y específicamente sobre los procesos cognitivos al amplificar los recursos mentales -se incrementa nuestra capacidad para codificar, almacenar, procesar y transmitir todo tipo de formación- y están cambiando nuestra forma de conocer (Adell, 1997; Bartolomé, 1999; Beltrán, 2001). También están cambiando los modos de interacción social:

- Por una parte, el volumen de la información disponible aumenta de forma exponencial. En determinados ámbitos del conocimiento, la información se duplica cada años. Ello supone la necesidad de actualización permanente y de diseñar y utilizar nuevos modos de organizar y acceder a la información (Adell, 1997). El hipertexto e Internet son caminos para ello, pero requieren destrezas de uso que hay que aprender. Por tanto, hay que trabajar el desarrollo de destrezas de acceso y selección de información más que la reproducción de conocimientos.

- Por otra parte, están los modos como se codifica la información. La información se ha almacenado durante siglos a través de la palabra escrita en forma de libros. Hoy esto está cambiando y el soporte de información evoluciona hacia sistemas multimedia, con un peso importante de la imagen e incluso del sonido (televisión, Internet...). Los cambios producidos por la televisión y los audiovisuales se pueden interpretar de forma negativa (superficialidad, pasividad, falta de espíritu crítico, irreflexión, dispersión de la atención, falta de estructuración del conocimiento, etc.) o en forma positiva, aceptando que potencian facetas de la actividad intelectual relacionadas con el hemisferio derecho (intuición, pensamiento global, representación visual, etc.). En este contexto, la escuela debe cambiar, no puede seguir tratando de transmitir el conocimiento como hace 50 años, basándose de forma casi exclusiva en la palabra, y especialmente en la palabra escrita.

- Una consecuencia importante de la ampliación de nuestra capacidad para procesar información derivada de las NT es la transformación radical de dos condicionantes fundamentales de la comunicación (Adell, 1997; Castells, 2000a): el espacio y el tiempo. Las nuevas tecnologías han desmaterializado y globalizado la información. Al situarla en el "ciberespacio" la han liberado de las características de los objetos 
culturales tradicionales (objetos muebles como el libro, el cuadro o la fotografía) que la sustentaban y cuya materialidad nos limitaba, y han eliminado los tiempos de espera para que el mensaje llegue del emisor al receptor. Las implicaciones de este cambio son importantísimas dado que las coordenadas espaciotemporales son el marco de la actividad humana.

- Por otra parte, si bien es cierto que las sucesivas revoluciones tecnológicas han alejado progresivamente al hombre de la biología y de la naturaleza, hay autores que destacan el carácter "natural" de los medios digitales. Levinson (1990) es uno de ellos: los nuevos medios electrónicos (analógicos primero y digitales después) no sólo extienden nuestras posibilidades de comunicación más allá de nuestros límites biológicos, sino que recuperan elementos y características de la etapa pretecnológica anterior a la escritura (interactividad entre emisor y receptor, tiempo real, uso directo de los sentidos, etc.). El uso de los artefactos nos ha devuelto los sentidos en la comunicación humana. Las nuevas tecnologías están haciendo un mundo más "natural"en el sentido antes indicado. Así se pronuncia Levinson. Evidentemente ésta es sólo una forma de verlo.

- Por fin, están las repercusiones de la interactividad. Frente a una masa indiferenciada, creada por los medios de comunicación tradicionales, aparecen comunidades "virtuales" más activas, que no sólo consumen información, sino que también la producen y distribuyen. Las redes informáticas permiten a los usuarios participar de nuevas de formas de interacción social. Así se crean esas comunidades virtuales, grupos de personas que comparten un interés y que utilizan las redes informáticas como canal de comunicación entre individuos espacialmente dispersos y temporalmente no sincronizados. La interactividad, junto con la deslocalización poseen implicaciones cruciales en todos los ámbitos de la experiencia. Así, por ejemplo, Internet puede soportar modelos pedagógicamente tradicionales de educación a distancia, pero están emergiendo nuevos entornos de enseñanza/aprendizaje basados no sólo en formas de comunicación en tiempo real (videoconferencia, por ejemplo), sino también en técnicas didácticas de aprendizaje cooperativo y colaborativo (Salinas, 1995). Estos entornos rompen la unidad de tiempo, espacio y actividad de enseñanza presencial, creando "aulas virtuales".

\section{La integración de las NTIC en la educación. Por una pedagogía activa y constructiva}

La introducción de las tecnologías de la información y la comunicación en educación y en el sistema educativo reglado no es tarea fácil. La escuela siempre va detrás de la sociedad y hay un desfase evidente entre el uso que de las NT se hace a nivel general, en las diversas actividades sociales y económicas cotidianas y el que se hace en la escuelas. Existen resistencias al cambio en la institución escolar, miedo a perder el control por parte de los profesores y falta de dominio de las nuevas tecnologías, dificultad para ajustar el rol tradicional a la nueva realidad, etc. Además, los cambios en este terreno se suceden a una velocidad vertiginosa, no dando demasiado tiempo para reflexionar sobre las posibilidades y consecuencias de su introducción en el aula. Todo ello ralentiza y dificulta la integración.

Sin embargo, lo cierto es que, paulatinamente, se va produciendo la introducción de las nuevas tecnologías, una introducción que induce cambios y que presenta ventajas en comparación con los recursos utilizados por la enseñanza tradicional (Adell, 1997, 1998; Bartolomé, 1999; Beltrán, 2001; Cabero, 1996 ; De Pablos, 1998; Joyanes, 1999; Kennedy, Odell y Klett, 2001):

- Flexibilidad instruccional (mediante las NT la enseñanza se puede adaptar a las 
posibilidades y necesidades individuales permitiendo una enseñanza más personalizada y el desarrollo de procesos de aprendizaje más constructivos y creativos).

- Complementariedad de códigos (la información multimedia llega por diversos canales sensoriales y se aprende mejor lo que se ve, se oye y se hace).

- Aumento de la motivación (los estudiantes se sienten más motivados haciendo uso de las NTIC).

- Desarrollo de actividades colaborativas y cooperativas (el uso adecuado de las TIC potencia el trabajo cooperativo entre los alumnos del grupo y también con otros a través de la red).

Y también riesgos e inconvenientes, que deben ser previstos para un uso óptimo. Entre ellos habría que destacar:

- La pseudoinformación (disponer de mucha información no significa estar más informado si no se ha dotado al sujeto de herramientas para seleccionar la información y para analizarla críticamente separando lo relevante de lo accesorio, tendencioso o manipulador).

- La saturación de la información (la sobrecarga de información puede producir un efecto de saturación cognitiva, que impediría el aprendizaje).

- La dependencia tecnológica (darle mayor valor al "saber cómo" que al "saber qué o por qué").

Para aprovechar las oportunidades y evitar los riesgos, nosotros postulamos una pedagogía de tipo cognitivista y constructivista (Hannafin, 1992; Hannafin, Land y Oliver, 2000; Jonassen, 2000) que integre las aportaciones la teoría socio-cultural (Cole, Vygotsky, Wertsch) (Beltrán, 2001). La información no es conocimiento, es el sustrato, la materia prima, necesaria pero no suficiente, y es la educación la que transforma la información en conocimiento. Se trata de arbitrar los medios pedagógicos necesarios para pasar de la "sociedad de la información" a la "sociedad del conocimiento", o, si se quiere, como dice Marina (1999), lograr que la "sociedad de la información" se traduzca en "sociedad del aprendizaje" y en "sociedad de la inteligencia".

¿Cuál es la ruta pedagógica adecuada para ello?. Desde nuestro punto de vista, una pedagogía constructivista, una pedagogía que utiliza estrategias para relacionar, combinar y transformar los conocimientos, una pedagogía que responde a un modelo de verdad a descubrir, centrado en la búsqueda, la indagación, la curiosidad, la exploración y la imaginación. Ello supone rediseñar la educación, lo que exige: (1) instaurar la "sociedad del aprendizaje" (Marina, 1999), el aprendizaje permanente, (2) replantear el papel del profesor -que ve reducida la exigencia de transmitir contenidos y que debe poner el énfasis en el diseño de situaciones instruccionales pertinentes ejerciendo una auténtica tutorización y seguimiento del proceso de aprendizaje del estudiante desde la perspectiva de la atención a la diversidad-, (3) revisar el papel del alumno -que debe aprender a ser el conductor de su propio proceso de aprendizaje, con la mediación necesaria del profesor, lo que exige adquirir las habilidades, destrezas y estrategias necesarias para el manejo de las nuevas tecnologías, para la toma de decisiones y para la elección de la propia ruta de aprendizaje-, (4) así como el de los contenidos de aprendizaje -el crecimiento exponencial del conocimiento fuerza a ser selectivos, enseñando lo que se puede y se debe aprender, no todo lo que se puede enseñar, y poniendo el énfasis en el aprender a aprender, en los procedimientos, en el saber hacer, en los modos de acceso y manejo de la información-, 
(5) utilizar nuevos materiales de aprendizaje (tutoriales multimedia, bases de datos online, bibliotecas electrónicas, simulaciones, hipertextos, etc.), y (6) reconsiderar también el papel del contexto -nuevos entornos de aprendizaje, nuevas "comunidades de aprendizaje" apoyadas en las nuevas tecnologías que favorecen la socialización del conocimiento, pasando de la construcción individual a la construcción social y que permiten ensayar nuevos modelos educativos que complementen los ya disponibles (Bereiter y Scardamalia, 1993; Means y Olson, 1995)-.

\section{Factores fundamentales para integrar las NTIC en la escuela}

Además de la pedagogía que postulamos, hay, desde nuestro punto de vista una serie de factores de gran importancia para una integración eficaz:

1. Apoyo institucional y desarrollo de políticas orientadas a la integración de las TIC en los centros, lo que comporta dotación de infraestructuras y formación del profesorado.

2. Apoyo de los centros y equipos directivos de los mismos a los proyectos de innovación pedagógica mediante las NT

3. Modelos de evaluación pertinentes y procesos de evaluación consecuentes.

1.- En la primera línea de acción son notables los esfuerzos desarrollados en Estados Unidos desde la administración Clinton (1993-2000) que puso en marcha en 1996 el primer plan nacional de tecnología educativa "Getting America's Students Ready for the 21 st Century", que incluye financiación, dotación de personal y formación de profesores. Para lograr las metas previstas se implementaron diversos programas, por parte del Gobierno Federal, entre los que destacan los siguientes: Technology Literacy Challenge Fund (TLCF) (http://www.ed.gov/ Technology/TLCF), Technology Innovation Challenge Grants Program (TICG) (http://www.ed.gov/ Technology/challenge), Star Schools Program (http://www.ed.gov/prog_info/ StarSchools/), Preparing Tomorrow's Teachers to Use Technology (PT3) (http://www.ed.gv.teachtech), y E-Rate Program (The Universal Service Fund for Schools and Librairies) (http://www.ed.gov/Technology/com-mit.html) (http://urban.org/education/ erate.html)

A partir del desarrollo de estos programas ha mejorado sustancialmente la ratio de alumnos por ordenador en los centros públicos (9'1 en 1993-94, 5'7 en 1999), así como la ratio de alumnos por ordenador conectado a Internet, que ha pasado, de 1998 a 1999, de 13 alumnos en primaria a 11 y de 10 en secundaria a 7 ( 7 para el total de centros de primaria y secundaria en 2000), también lo ha hecho la conexión a Internet en las escuelas (30\% de las escuelas públicas de nivel elemental en 1994 frente al $94 \%$ en 1999 y $49 \%$ en los centros públicos de secundaria frente al $98 \%$ de 1999; 98\% para el total de los centros en 2000) (CEO Forum, 2001; National Center for Education Statistics, 2000a, 2000 b).

La Unión Europea también trabaja en esta misma línea. Algunos documentos clave son los siguientes: el informe "Europa y la sociedad global de la información: Recomendaciones al Consejo Europeo" (http://xina.uoc.es:443/docs_elec/2704.htm), conocido como informe Bangeman, publicado en 1994, el "Plan de actuación. Europa en marcha hacia la sociedad de la información" (Europe's way to the Information Society) (Comisión de las Comunidades Europeas, 1994), el "Libro Blanco sobre la Educación y la Formación" (Comisión de las Comunidades Europeas, 1995), el "Libro Verde" ("Green Paper. Living and working in the information society") (Comisión de las Comunidades Europeas, 1996a), el documento "Aprendizaje en la sociedad de la información. Plan de acciones para una iniciativa europea en educación (1996-98)", también de 1996, el informe de la Comisión al Consejo y al Parlamento Europeo "Concebir la Educación del Futuro. Promover la innovación con las nuevas tecnologías" (Comisión de las 
Comunidades Europeas, 2000a), la Comunicación de la Comisión "Estrategias para la creación de empleo en la sociedad de la información" (Comisión de las Comunidades de las Comunidades Europea, 2000b), y el "Memorándum sobre el aprendizaje permanente" (2000e).

Desarrollos concretos a partir de estas directivas son The European Schoolnet (http://www.eun.org), la red europea de escuelas, creada en 1997, la semana de los Netd@ys (http://europa.eu.int/comm/education/netdays/index.html), la iniciativa "eEurope" (1999), con la adopción por parte de la Comisión de la Comunicación "eEurope. Una Sociedad de la Información para todos" (http://europa.eu.int/comm/information_society/eeurope/documentation/index_ en.htm), que fue aprobada por el Consejo y por el Parlamento Europeo (Comisión Europea, 2000c) en el Consejo Extraordinario de Lisboa, y la iniciativa "eLearning", también de 2000 (Comisión Europea, 2000d), que articula los diferentes componentes de las acciones de eEurope. A ello se añade la acción Minerva del Programa Sócrates II (http:://europa.eu.int/comm/education/socrates/minerva/ind1a.html).

Las líneas prioritarias de acción son:

- Equipamiento (todas las escuelas de la Unión Europea deben tener acceso a Internet y a los recursos multimedia antes de finales de 2001; y antes de finales de 2004 la ratio de usuarios por ordenador multimedia en las escuelas debe estar entre 5-15)

- Formación (de profesores -antes de finales de 2002 todos deben estar capacitados para usar Internet y los recursos multimedia- y alumnos -antes de finalizar 2003 todos deben tener una "formación digital" al dejar las aulas)

- Desarrollo de servicios y contenidos multimedia de calidad

- Desarrollo e interconexión de centros educativos y científicos.

- Enseñanza a distancia.

Con todo ello, la situación en Europa es desigual: hay países que se acercan al nivel de EEUU (como Dinamarca, Finlandia o Suecia) y otros que se encuentran mucho más lejanos, como ocurre con el nuestro, que está en la zona baja de la UE (ISPO, 2000).

En España, además de los programas comunitarios se están llevando a cabo proyectos a nivel nacional y autonómico, de cara a potenciar la integración de las NTIC en los procesos formativos.

El Ministerio de Educación y Ciencia puso en marcha en noviembre de 1989 el "Programa de Nuevas Tecnologías de la Información y la Comunicación" (PNTIC) (http://www.pntic.mec.es), que desarrolló el Proyecto Atenea y el Proyecto Ágora (http://www.pntic.mec.es/ main_agora.html).

El PNTIC ha sido sustituido por el Centro Nacional de Información y Comunicación educativa (CNICE) (http://www.cnice.mecd.es/), creado en abril de 2000, dependiente del actual Ministerio de Educación, Cultura y Deporte, que mantiene tres proyectos en desarrollo: Aldea Digital (http://www.cnice.mecd.es/Aldea_Digital/aldea.html), Aulas Mentor (http://www. mentor.mec.es/d_marco2.htm) y Aulas Hospitalarias (http://www.cnice.mecd.es/ proyectos/aulashosp/proyecto.htm).

El sistema descentralizado español ha comportado que sean las administraciones autonómicas las que hayan ido asumiendo las competencias, los retos y las obligaciones en el terreno que nos ocupa. Aunque existen diferencias entre las Comunidades, debido al distinto momento en que han recibido las competencias educativas del Estado, las actuaciones que están realizando en este terreno afrontan los tres elementos 
fundamentales en los que se asienta la introducción y uso de las NTIC en el aula:

- Dotación de infraestructuras

- Formación técnica y pedagógica del profesorado

- Servicios de gestión y recursos educativos

La formación de profesores es un elemento fundamental dentro de las políticas para la integración de las NTIC. Como en cualquier innovación que se quiera introducir en la escuela, el profesor es el eje central del proceso. Como se demuestra en diversos estudios, no basta con dotar a los centros con recursos tecnológicos, dado que es el profesor el que, en función de su motivación, intereses y formación, se convierte en el protagonista principal, en motor del cambio. No se trata de algo específico de los programas de introducción y uso de las NTIC, sino de una constante en cualquier programa de innovación.

Así, en EEUU, que ha hecho de la introducción de las TIC y específicamente de Internet una cuestión nacional que aglutina esfuerzos de todas las administraciones y de la iniciativa privada, se han desarrollado programas específicos de formación de profesores, como es el programa PT3 (Preparing Tomorrow's Teachers to Use Technology) (http://www.ed.gov/teachtech), dirigido a la preparación de los futuros profesores. Se trata de un programa federal implementado en toda la nación desde 1999.

Se han desarrollado también multitud de iniciativas para la formación de profesores en ejercicio por parte de la administración, de las universidades y de entidades privadas. Un ejemplo son los cursos web de la Universidad de Idaho para capacitar a los maestros (http://ivc.idaho.edu) (Kennedy, Odell y Klett, 2001).

La Unión Europea también considera la formación de profesores como un eje fundamental de las políticas de integración de las TIC y así se refleja en diversos documentos, planes e iniciativas de los que ya hablamos antes.

En nuestro país el planteamiento es similar y la formación de los profesores ha sido siempre una de las prioridades, tanto de las iniciativas desarrolladas por el MEC a través del PNTIC y del CNICE como de las desarrolladas por las Comunidades Autónomas (Infocole, Xarxipèlago, XTEC, Averroes, etc.)

Hay diversos estudios que confirman que no es suficiente dotar a los centros de las herramientas informáticas, sino que es necesario que los profesionales del ámbito educativo se impliquen en el proceso. Para ello, es imprescindible conocer la utilidad pedagógica de los recursos tecnológicos, tanto a nivel teórico como práctico, y disponer de ayudas institucionales que favorezcan o fomenten la integración de estos recursos. Es necesaria una "alfabetización" tecnológica de los profesores pero también formación en uso "educativo" de la red.

2.- Como se demuestra en algunos estudios realizados, el uso del ordenador en el proceso de enseñanza-aprendizaje está relacionado con el interés de la escuela (directivos y profesores) por la tecnología. Equipos directivos dinámicos y proclives a la innovación favorecen y facilitan la introducción de las TIC (Gargallo, Suárez, Morant, Marín, Martínez y Díaz, 2002, en prensa).

Los centros pueden facilitar el proceso de integración con diferentes acciones:

- Disponiendo la infraestructura necesaria para la implementación generalizada de las NTIC en el proceso educativo.

- Ejerciendo una labor de dinamización y coordinación 
- Propiciando que los profesores dispongan del tiempo necesario para integrar en su docencia la tecnología aplicándola a los procesos de enseñanza-aprendizaje.

- Creando comisiones de tecnología, formadas por varios profesores motivados en relación con su uso en la enseñanza.

Dado que el proceso de integración puede resultar difícil y arduo, es necesario que los profesores cuenten en todo momento con el apoyo del equipo directivo de su centro, con el fin de facilitar en lo posible el trabajo a realizar. Las barreras para la introducción de las TIC son tanto técnicas como sociológicas e institucionales.

3.- La evaluación del impacto de las nuevas tecnologías en la educación reviste una gran complejidad y presenta notables dificultades dado que esas tecnologías son muy diversas, afectan a todo el escenario escolar y es difícil controlar los efectos inducidos en todos los ámbitos en que se utilizan las NTIC (administración, procesos de enseñanza-aprendizaje, organización, formación de profesores, etc.). Por otra parte, no es fácil evaluar sus efectos en la educación dado que la tecnología no es generalmente una causa directa sino más bien un facilitador o amplificador de las diversas prácticas educativas (Lesgold, 2000). Además, el contexto en que se inserta la tecnología es fundamental de cara a su uso eficaz: infraestructuras, cultura escolar, actitudes de los profesores y su dominio del manejo tecnológico, que es muy variable, equipos más o menos estables y comprometidos, modelos organizativos, etc., mediatizan sus efectos. Un centro muy bien dotado puede realizar un uso mediocre de la tecnología mientras que un centro con muy pocos recursos puede hacerlo de manera brillante. Todo ello nos hace volver a la idea de complejidad que enunciamos al principio y nos incita a ser cautos: es difícil determinar los efectos de la tecnología en contextos complejos y con referencia a habilidades complejas. Decimos esto último porque muchas de las propuestas de integración y uso en las escuelas se abordan desde postulados constructivistas, y trabajan para desarrollar habilidades complejas en los estudiantes: solución de problemas, pensamiento creativo, producción de ideas, reconstrucción de las teorías, planificación, adquisición, análisis y uso de la información, colaboración, etc. y las habilidades complejas no se evalúan bien con tests estandarizados (Haertel y Means, 2000), que evalúan más bien habilidades básicas.

Y, sin embargo, es necesario afrontar de modo sistemático la evaluación de los efectos de las NTIC para determinar su verdadero papel y dado que se dedican recursos importantes a su introducción en la escuela y es necesario aprovecharlos y optimizar el proceso.

Problemas complejos requieren de métodos complejos. Desde nuestro punto de vista, un acercamiento adecuado al tema de la evaluación del impacto de las NTIC precisa de planteamientos integradores, de métodos cuantitativos y cualitativos (Rumberger, 2000), de evaluación formativa -más sensible al contexto y a los intereses de los implicados- y sumativa -que generalmente goza de mayor rigor técnico aunque es menos sensible al contexto- (Baker y Herman, 2000).

Estos planteamientos integradores son evidentes en diversos trabajos de evaluación realizados en este contexto, referidos a proyectos de amplio calado, que pueden resultar ejemplares: así la evaluación del proyecto PT3 (Preparing Tomorrow's Teachers to Use Technology), un proyecto incluido en el primer plan nacional de tecnología educativa del gobierno americano, para formar a los futuros profesores en su uso (http://www.ed.gov/teachtech), que incluye elementos cuantitativos y cualitativos. En la misma línea se orienta la evaluación del Programa OCDE/CERI sobre TIC y calidad del aprendizaje (CERI Programa on ICT and the Quality of Learning). Se trata de un programa iniciado en 1998 por CERI (Centre for Educational Research and Innovation) bajo los auspicios de la OCDE. La evaluación se ha abordado a través de "estudios de caso" y de "estudios cuasiexperimentales" (OCDE, 2000a, b y c). 
Además de planteamientos integradores, la evaluación en este campo requiere precisión en las metas y objetivos y disponibilidad de estándares e indicadores -prácticamente inexistentes en lo referido a la integración de Internet en la escuela en el ámbito europeo y español-, lo que reclama un trabajo riguroso en los próximos años para concretarlos.

Por otra parte, una condición necesaria, sobre todo en investigaciones y evaluaciones referidas a programas de envergadura, nacionales, autonómicos, etc. que necesitan apoyo institucional para su desarrollo, es la delimitación de los órganos responsables de su implementación.

En Estados Unidos, la Oficina de Tecnología Educativa del Departamento de Educación (http://www.ed.gov/Technology) desarrolla la política educativa nacional en el campo delas NTIC y la implementa a través de los diversos programas, y su Servicio de Planificación y Evaluación (PES: Planning and Evaluation Service) (http://www.ed.gov/offices/OUS/PES/edtech.html) evalúa la eficacia de los programas federales de tecnología educativa. Todo ello hace que la evaluación sea un sector fundamental en el que también este país es puntero. Las diversas actividades de evaluación pueden ser consultadas en la web del Departamento de Educación (http://www.ed.gov/Technology/ eval_activities.html)

En la Unión Europea la situación es más difusa dado que muchas de las propuestas son directivas y recomendaciones para los países integrantes, que son quienes tienen que implementarlas. La UE aporta fondos, orientaciones y dispone un calendario de ejecución, como hemos visto. No tenemos conocimiento de que exista un órgano o departamento dedicado a la evaluación de programas de este tipo.

En nuestro país hay órganos de la administración cuyo cometido es la evaluación del sistema (INCE a nivel nacional, Institutos y organismos similares creados en algunas comunidades autónomas: IVECE en Valencia, Consell Superior d'Avaluació en Catalunya, ICEC en Canarias, etc, y Unidades de Evaluación de las Comunidades que todavía no han creado un órgano específico de evaluación de esta índole). Sin embargo, ni las unidades de evaluación de las Comunidades Autónomas, ni los Institutos u órganos específicos de evaluación del sistema educativo, ni tampoco los programas de nuevas tecnologías de las administraciones autonómicas han realizado, hasta el momento, una evaluación sistemática del uso de las NTIC y de sus repercusiones en la educación. Generalmente se limitan a recoger periódicamente datos de dotaciones y recursos disponibles en los centros. Ello tiene su lógica, dado que la prioridad ha sido la dotación, que, como ya dijimos antes, se encontraba y se encuentra todavía en cotas muy bajas comparándonos con otros países de la UE, y, por supuesto, con EEUU. Desde nuestro punto de vista, la evaluación del impacto de las NTIC en el sistema educativo español es una de las cuestiones fundamentales a abordar en los próximos años, tanto a nivel macro como a nivel micro, para precisar su incidencia en nuestros centros escolares a nivel organizativo, a nivel de procesos de enseñanza y a nivel de resultados y procesos de aprendizaje, y ahí las instituciones diseñadas para la evaluación del sistema tienen un papel fundamental [1].

\section{Bibliografía}

ADELL, J. (1997) Tendencias en educación en la sociedad de las tecnologías de la información, Edutec, 7, http://www.uib.es/depart/gte/revelec7.html

ADELL, J. (1998) Redes y educación, en DE PABLOS, J. y JIMÉNEZ, J. (eds.) Nuevas Tecnologías, Comunicación Audiovisual y Educación. Barcelona, Cedecs.

AIMC (2001) Estudio General de Medios.


BAKER, E.L. y HERMAN, J. L. (2000) Technology and Evaluation. Trabajo presentado en el Meeting realizado en SRI International dentro del proyecto "Building Foundation for a Decade of Rigorous, Systematic Educational Technology Research", subvencionado por el Departamento de Educación de EEUU. Menlo Park, California, Febrero. http://www.sri.com/policy/designkt/found.html

BANGEMANN, M. (1994) Europa y la sociedad global de la información: recomendaciones al Consejo Europeo. Bruselas, 24 de mayo de 1994. (http://xina.uoc.es:443/docs_elec/2704.htm)

BARBERÁ, J. (1995) Veinticinco años de Internet: una retrospectiva autobiográfica, Boletín de RedIRIS, 32, julio de 1995. Versión electrónica en: http://www.rediris.es/rediris/boletin/32/enfoque2.html

BARTOLOMÉ, A. R. (1999) Las nuevas tecnologías y la educación, en MARINA, J. A. et. al. Educación e Internet. Documentos del $1^{\circ}$ Congreso Educación e Internet. Educnet 99. Madrid, Santillana.

BELTRÁN, J. (2001) La nueva pedagogía a través de Internet, en I Congreso Nacional de Educared. Madrid, 18-20 de enero. Documento electrónico: http://www.educared.net/htm/congreso-i/documentacion.htm

BEREITER, C. y SCARDAMALIA, M. (1993) Enfoques de primero, segundo y tercer orden para mejorar las estrategias cognitivas de aprendizaje de la escritura, en BELTRÁN, J. et al. Intervención psicopedagógica. Madrid, Pirámide.

BOSCO, J. (1995) Schooling and Learning in an Information Society, en U. S. Congress, Office of Technology Assessment, Education and Technology: Future Versions, OTA-BPHER-169. Washington DC, Government Printing Office

CABERO, J. (1996) Nuevas tecnologías, comunicación y educación, Edutec, 1. Documento electrónico: http://www.uib.es/depart/gte/revelec1.html

CASTELLS, M. (2000a) La era de la información. Vol. 1. La sociedad red. (2a edición). Madrid, Alianza.

(2000b) Internet y la Sociedad Red. Lección inaugural del programa de doctorado sobre la sociedad de la información y el conocimiento. Universitat Oberta de Catalunya. Documento electrónico: http://www.uoc.es/web/esp/articles/castells/print.html CEO Forum (2001) School Technology and Readiness Report. Key Building Blocks for Student Achievement in the 21st Century: Assessment, Alignment, Accountability, Access, Analysis. Star Four. Documento electrónico: http://www.ceoforum.org/reports.cfm COMISIÓN DE LAS COMUNIDADES EUROPEAS (1995) White paper on education and training. Teaching and Learning. Towards the learning society. Luxemburgo, Oficina de publicaciones oficiales de las Comunidades Europeas (http://europa.eu.int/comm/education/lb-en.pdf)

COMISIÓN DE LAS COMUNIDADES EUROPEAS (1996a) Green Paper. Living and Working in the Information Society: People First. Documento electrónico: http.//www.hamburg.de/ English/StadtPol/Europe/peopl1st.htm

COMISIÓN DE LAS COMUNIDADES EUROPEAS (1996b) Learning in the Information Society. Actions plan for a European education initiative (1996-98). Documento electrónico: http://europa.eu.int/comm/education/elearning/eplanen.pdf COMISIÓN DE LAS COMUNIDADES EUROPEAS (2000a) Concebir la educación del futuro. Promover la innovación con las nuevas tecnologías. Documento electrónico: http://europa.eu.int/comm/education/elearning/rapes.pdf

COMISIÓN DE LAS COMUNIDADES EUROPEAS (2000b) Estrategias para la creación de empleo en la sociedad de la información. Documento electrónico: http://europa.eu.int/comm/employment social/soc-dial/info-soc/news/es.pdf

COMISIÓN DE LAS COMUNIDADES EUROPEAS (2000c) eEurope. Una Sociedad de la Información para todos. Documento electrónico: http://europa.eu.int/comm/information_society/eeurope/documentation/index_en.htm COMISIÓN DE LAS COMUNIDADES EUROPEAS (2000d) eLearning-Concebir la educación del futuro.

Documento electrónico: 
http://europa.eu.int/comm/education/elearning/index.html

COMISIÓN DE LAS COMUNIDADES EUROPEAS (2000e) Memorándum sobre el aprendizaje permanente Documento electrónico:

http:europa.eu.int/comm/education/life/guide_es.pdf

DE PABLOS, J. (1998) Nuevas tecnologías aplicadas a la educación: una vía para la innovación, en DE PABLOS, J. y JIMÉNEZ, J. (eds.) Nuevas Tecnologías, Comunicación Audiovisual y Educación. Barcelona, Cedecs.

ECHEVARRÍA, J. (1994) Telépolis. Barcelona, Anagrama.

EISENSTEIN, E. (1994) La revolución de la imprenta en la edad moderna. Madrid, Akal.

GALLEGO, J. (2001) Internet: estrategias para una innovación educativa. Ponencia presentada en I Congreso Nacional de Educared. Madrid, 18-20 de enero. Documento electrónico: http://www.educared.net/htm/congreso-i/documentacion.htm

GARCÍA CARRASO et. al. (1998) Espacios reales, espacios virtuales, espacios educacionales universitarios, en XVII Seminario Interuniversitario de Teoría de la Educación. Málaga, 16-18 de noviembre.

GARGALLO,B., et. al. (2002) Un primer diagnóstico del uso de Internet en los centros escolares de la Comunidad Valenciana. Procesos de formación y efectos sobre la calidad de la educación. Valencia. Conselleria de Cultura i Educació, IVECE. En prensa.

GATES, B. (1995) Camino al futuro. Madrid, McGraw-Hill.

HAERTEL, G. y MEANS, B. (2000) Stronger Designs for Research on Educational Uses of Technology: Conclusion and Implications. Documento electrónico: http://www.sri.com/policy/designkt/found.html

HANNAFIN, M. J. (1992) Emerging technologies, ISD, and learning environments: critical perspectives, Educational Technology Research and Development, 40 (1), 49-63.

HANNAFIN, M. J., LAND S. y OLIVER, K. (2000) Entornos de aprendizaje abiertos: fundamentos, métodos y modelos, en REIGELUTH, CH. M. (ed.): Diseño de la instrucción. Teorías y modelos. Un nuevo paradigma de la teoría de la instrucción. Parte I. Madrid, Aula XXI/Santillana.

HARNAD, S. (1991) Post-Guttemberg Galaxy: The Fourth Revolution in the Means of Production of Knowledge, The Public-Access Computer System Review, 2 (1), 39-53.

JONASSEN, D.H. (2000) El diseño de entornos constructivistas de aprendizaje, en REIGELUTH, CH. M. (ed.) Diseño de la instrucción. Teorías y modelos. Un nuevo paradigma de la teoría de la instrucción. Madrid, Aula XXI/Santillana.

JOYANES, L. (1996) Cibersociedad: Realidad o Utopía. Madrid, UPSA.

(1999) El nuevo perfil social y cultural de la era Internet: la sociedad del conocimiento, en MARINA J. A. et. al. Educación e Internet. Documentos del $1^{\circ}$ Congreso Educación e Internet. Educnet 99. Madrid, Santillana.

KENNEDY, T.J., ODELL, M.R.L. y KLETT, M.D. (2001) Internet en las escuelas de Estados Unidos: Una perspectiva desde el programa GLOBE, en I Congreso Nacional de Educared. Madrid, 18-20 de enero. Documento electrónico: http://www.educared.net/htm/congreso-i/documentacion.htm

LESGOLD, A. (2000) Determining the Effects of Technology in Complex School Environments. Paper Comissioned by SRI International for the United States Department of Education. Trabajo presentado en el Meeting realizado en SRI International dentro del proyecto "Building Foundation for a Decade of Rirgorous, Systematic Educational Technology Research", subvencionado por el Departamento de Educación de EEUU. Menlo Park, California, Febrero. Documento electrónico: http://www.sri.com/policy/designkt/found.html

LEVINSON, P. (1990) Computer Conferencing in the Context of the Evolutions of Media, en HARASIM, L. M. Online Education. Perspectives on a New Environment. Nueva York, Praeger Press.

MARINA, J.A. (1999) El timo de la sociedad de la información, en MARINA, J. A. et. al. Educación e Internet. Documentos del $1^{\circ}$ Congreso Educación e Internet. Educnet 99. 
Madrid, Santillana.

MEANS, B. y OLSON, K. (1995) Technology's role within constructivist classrooms, en Annual Meeting of the American Educational Research Association. San Francisco.

NATIONAL CENTER FOR EDUCATION STATISTICS (2000a) Internet Access in U.S. Public Schools and Clasrooms: 1994-99. NCES 2000-086. Education Publications Center (ED Pubs). U.S. Department of Education, Washington.

NATIONAL CENTER FOR EDUCATION STATISTICS (2000b) Teacher Use of Computers and the Internet in Public Schools. NCES 2000-090. Education Publications Center (ED Pubs). U.S. Department of Education, Washington.

NEGROPONTE, N. (1995) El mundo digital. Barcelona, Ediciones B.

OCDE (2000a) Methodology for Case Studies of Organisational Change. Documento electrónico: http://bert.eds.udel.edu/oecd/cases/CASES11.html

OCDE (2000b) Estudio de casos sobre el cambio en la organización escolar. Cuaderno de trabajo. OECD/CERI SEP/ILCE-UPN. México. Documento electrónico: http://bert.eds.udel.edu/oecd/cases/wrkbkSpanish.doc

OCDE (2000c) The impact of ICT on learning: design for a quasi-experimental study Documento electrónico: http://bert.eds.udel.edu/oecd/experiments/experimentshome.html ONG, W.J. (1995) Orality \& Literacy: The Technologizing of the World. Londres, Routledge.

POSTMAN, M. (1994) Tecnópolis. Barcelona, Círculo de Lectores.

RUMBERGER, R.W. (2000) A Multi-level, Longitudinal Approach to Evaluating the Effectiveness of Educational Technology. Trabajo presentado en el Meeting realizado en SRI International dentro del proyecto "Building Foundation for a Decade of Rirgorous, Systematic Educational Technology Research", subvencionado por el Departamento de Educación de EEUU. California, Menlo Park. Documento electrónico: http://www.sri.com/policy/designkt/found.html

SANZ, M. A. (1994) ABC de Internet, Boletín de RedIRIS, 28. Versión electrónica en http://www.rediris.es/rediris/boletin/28/enfoque1.html

STOLL, C. (1996) Silicon Snake Oil: Second Thoughts on the Information Higway. Ancor Books.

TERCEIRO, J. B. (1996) Sociedad digital. Madrid, Alianza.

\section{Notas}

[1].- El Programa de Nuevas Tecnologías (PNTIC) del MEC, el de más tradición en nuestro país, ya desaparecido y sustituido por el CNICE antes mencionado, ha llevado a cabo evaluaciones de los diferentes proyectos que ha cobijado: en ocasiones han sido encargadas a evaluadores externos, como es el caso del Proyecto Atenea, cuya fase experimental fue evaluada por la OCDE, o como es el caso del Proyecto Mentor, actualmente en vigor; evaluado en su fase experimental por ISDEFE (Ingeniería de Sistemas) a través de cuestionarios pasados a tutores, alcaldes, administradores y alumnos, entrevistas a administradores, tutores y alcaldes, y observación "in situ" (ISDEFE, 2001) con un enfoque predominantemente cuantitativo. La transferencia de las competencias educativas a las diversas Comunidades Autónomas, ya completada, vuelca sobre ellas la responsabilidad de este tipo de evaluaciones, independientemente de la coordinación o iniciativas que pueda desarrollar el INCE (Instituto Nacional de Calidad y Evaluación). 